\title{
北极地区表层海水中持久性有机污染物 和重金属污染的现状
}

\author{
姚子伟 ${ }^{(1)}$ 江桂斌 ${ }^{(1 *}$ 蔡亚歧 $^{(1)}$ 徐恒振 ${ }^{(2)}$ 马永安 ${ }^{(2)}$
}

(1)中国科学院生态环境研究中心, 北京 100085; (2)国家海洋环境监测中心, 大连 116023.* 联系人: E-mail: gbjiang@ mail.rcees.ac.cn)

摘要 利用毛细管气相色谱-微池电子捕获检测器 (GC- $\mu$ ECD) 和气相色谱-质谱法(GC-MS) 研究测定了 北极地区表层海水中的含氯有机化合物的污染状况. 与前人的结果相比, 有机氯农药含量已大为降低. 白令海及楚科奇海区有机氯化合物总量基本相同，但组成不同. $\alpha / \gamma$-六六六 $(\mathrm{HCHs})$ 的比值可以作为推断 其来源及海流情况的标记. 利用电感耦合等离子体质谱(ICP-MS)分析考察了海水中的痕量重金属. 结 果表明: 该海区基本不存在重金属污染，海水中不同种类重金属呈现出不同的垂直分布状况.

\section{关键词 北极地区 表层海水 持久性有机污染物 重金属}

持久性有机污染物 (POPs) 和重金属是环境中常 见的污染物 ${ }^{[1]}$. 它们普遍存在于各种环境介质中, 大 多数具有致癌、致畸、致突变效应, 易在生物体内富 集并通过食物链放大, 最终对人体产生危害. 大量的 研究表明, 此类物质可以通过大气或海流的运输而 散布到整个世界, 而北极地区已经成为 POPs 的汇集 源, 尤其是以 $\mathrm{HCHs}$ 为代表的有机氯农药的含量大大 高于人们的估计. 主要原因是通过大气的长途输送 而汇集, 而由于极地地区的低温使海水中有机物难 以转化或降解 ${ }^{[2]}$. 最新的研究表明北极地区大气中的 $\mathrm{HCHs}$ 含量急剧下降, 导致海-气交换发生逆转, 表层 海水中的 $\mathrm{HCHs}$ 重新挥发到大气中, 使该地区成为 $\mathrm{HCHs}$ 的二次污染源. 这些研究主要集中在北太平洋 及格陵兰岛地区, 而白令海及楚科奇海地区仅有非 常有限的资料 ${ }^{[3 \sim 9]}$.

1999年 7月至 9 月间, 中国政府组织了第一次针 对楚科奇海和白令海的大规模的综合考察. 这次考 察的一个重要任务就是调查此海区的重金属及持久 性有机污染物的分布状况, 进而评价其对环境的影 响. 本文作者之一参加了这次考察活动并采集了大 量样品, 利用气相色谱、气相色谱-质谱联用(GC-MS) 以及电感耦合等离子体质谱(ICP-MS)等技术手段, 研究了白令海及楚科奇海地区的表层海水中痕量污 染物的含量和分布特征.

\section{1 实验}

(i ) 站位及样品采集. (1) 站位. 所有样品都是 在 1999 年 7 月至 9 月的中国首次北极科学考察期间
采集的. 样品站位示意图见图 1. 站位的选择主要从 水文学及地质学的角度考虑. 白令海区域站位覆盖 了约 240000 平方公里的开放区域, 基本上涵盖了从 陆架到陆坡及深海的范围. 楚科奇海站位既包括开 阔水域又有浮冰区, 表层浮冰比例从 $1 \%$ 到近 $80 \%$.

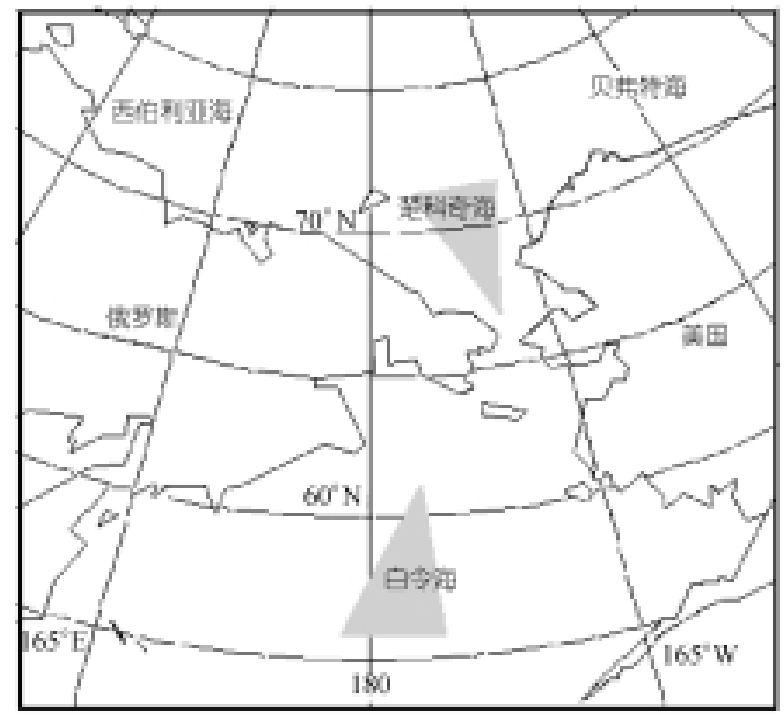

图 1 采样区域示意图 图中阴影部分为采样区域

(2) 采样. 为了避免船体的污染, 所有的样品在 船未完全停稳之前在迎风的一侧采集, 或根据船载 多普勒海流计的数据在冲着海流的方向. 采样前采 样器、绳子及样品瓶都经过蒸馏水洗涤, 并用现场海 水洗涤 2 次以上. 表层重金属样品用聚乙烯瓶采集, 分析有杋物的样品用玻璃瓶采集. 用与温度-盐度-深 
度探头 (CTD)连在一起的内壁为 Teflon 材料的梅花型 采水器(General Oceanics, 美国)采集分层水样.

（3）样品处理. 重金属样品采集后立即用 MOS 级硝酸调节 $\mathrm{pH}$ 值 2 左右. 有机物水样在船上进行液 液萃取, 一般每个站位采集 $4 \mathrm{~L}$ 表层水样, 每 $1 \mathrm{~L}$ 用 $50 \mathrm{~mL}$ 二氯甲烷萃取 2 次, 合并萃取液并于 $4^{\circ} \mathrm{C}$ 以下 避光保存在棕色样品瓶中. 萃取液在实验室中加人 适量无水硫酸钠除水后, 用旋转蒸发器浓缩并重新 溶解于 $5 \mathrm{~mL}$ 正己烷中, 用氮气浓缩定容至 $0.2 \mathrm{~mL}$, 取 $1 \mu \mathrm{L}$ 进样.

(ii) 标准及试剂. 有机氯农药(OCPs)和多氯联 苯 $(\mathrm{PCB})$ 混合标准购自 ChemService 公司 (West Chester, USA), OCPs 包括: $\alpha-, \beta-, \gamma$-及 $\delta$ - $\mathrm{HCH}$, 七氯, 环氧七氯, 艾氏剂, 硫丹 I, 硫丹 II, 硫丹硫酸盐, 狄氏剂, 异狄氏剂, 异狄氏剂乙醛, p,p'-DDE, p,p'-DDD 和 p,p'-DDT. PCBs 混合标准包括: CB28, CB52, CB101, CB112, CB118, CB138, CB153, CB155, CB180 和 CB198(均按 IUPAC 序号). 重金属标准为安 捷伦公司 ICP-MS 环境分析标准溶液.

储备液用甲苯和正己烷 $(1 ： 1)$ 配成, 浓度为 20 $\mu \mathrm{g} / \mathrm{mL}$, 在冰箱中避光保存. 所有试剂均为分析纯, 使用前经过重蒸处理. 所有玻璃器血均用丙酮和正 己烷冲洗. 在实验开始阶段做试剂空白. 色谱图上无 干扰峰出现.

(iii) 仪器条件. (1) GC- $\mu$ ECD. 气相色谱为带有 电子压力控制装置的 HP 6890A 气相色谱仪, 配 ${ }^{63} \mathrm{Ni}$ 微池电子捕获检测器 ( $\mu$-ECD). 色谱柱为 $30 \mathrm{~m}$ 长, 内 径 $0.25 \mathrm{~mm}$ 的 HP-1 熔融石英毛细管柱, 液膜厚度为 $0.25 \mu \mathrm{m}$. 升温程序: 起始温度 $150^{\circ} \mathrm{C}$ (保持 $2 \mathrm{~min}$ ), 以 $4^{\circ} \mathrm{C} / \mathrm{min}$ 的速率升温至 $270^{\circ} \mathrm{C}$ (保持 $10 \mathrm{~min}$ ); 载气和 检测器补充气为高纯氮气 $(99.99 \%)$, 柱流量为 1 $\mathrm{mL} / \mathrm{min}$, 补充气流量为 $60 \mathrm{~mL} / \mathrm{min}$. 进样口温度为 $250{ }^{\circ} \mathrm{C}$, 不分流进样模式, 检测器温度为 $300^{\circ} \mathrm{C}$.

(2) GC-MS. GC-17A/MS-5050A(岛津公司, 日 本), 色谱柱为 DB-1(30 m 长, 内径 $0.25 \mathrm{~mm}$, 液膜厚 度 $0.25 \mu \mathrm{m})$. 载气为氦气, 柱流量为 $1 \mathrm{~mL} / \mathrm{min}$, 不分 流进样, 进样口温度为 $250^{\circ} \mathrm{C}$, 温度程序与 GC- $\mu \mathrm{ECD}$ 方法相同, 气相色谱与质谱仪连接的界面温度为 $230^{\circ} \mathrm{C}$, 电子轰击源温度为 $250^{\circ} \mathrm{C}$. 质谱部分在全扫 描模式时扫描范围为 50 500 ( m/z).

(3) ICP-MS. Agilent 7500 ICP-MS(安捷伦公司, 美国). 仪器操作参数: 正相功率, $1350 \mathrm{~W}$; 喷雾器气
体流速, $1.2 \mathrm{~mL} / \mathrm{min}$; 采样深度, $6 \mathrm{~mm}$; 质谱为全质 量数扫描模式.

\section{2 结果与讨论}

\section{1 方法评价}

根据对比实际样品和标准的 GC- $\mu \mathrm{ECD}$ 谱图的保 留时间以及 GC-MS 的质谱图定性, 外标法定量分析 OCPs 和 PCBs, 它们在 1 1000 pg/L 范围内保持线性 关系 $\left(r^{2} \geqslant 0.998\right)$. OCPs 回收率为 $59 \% \sim 92 \%, \mathrm{PCBs}$ 回 收率在 $85 \%$ 111\%. OCPs 的方法检出限从 $0.7 \mathrm{pg} \cdot \mathrm{L}^{-1}$ (p,p'-DDD)到 $27 \mathrm{pg} \cdot \mathrm{L}^{-1}$ (p,p'-DDT). PCBs 的方法检 出限为 $1.9 \mathrm{pg} \cdot \mathrm{L}^{-1}(\mathrm{CB} 112)$ 到 $3.2 \mathrm{pg} \cdot \mathrm{L}^{-1}$ (CB52). 重 金属分析中, 由于 Agilent 7500 ICP-MS 具有高盐防 堵喷头, 海水样品未经过滤直接进样, 以 ${ }^{209} \mathrm{Bi}$ 为内 标定量.

\section{2 白令海及楚科奇海表层海水中 OCPs 的分布特 征:}

(1) 污染状况及污染物组成. $\mathrm{HCHs}$ 是世界范 围广泛存在的污染物. 本次考察中, 白令海区的 24 个站位中有 22 个检出了 $\alpha-\mathrm{HCH}$, 最高浓度为 608 $\mathrm{pg} \cdot \mathrm{L}^{-1}$; 在楚科奇海的 15 个站位中有 14 个检出, 最 高浓度为 $641 \mathrm{pg} \cdot \mathrm{L}^{-1}$. 相关文献表明 ${ }^{[4,6,7]}$, 海水中的 $\mathrm{HCHs}$ 浓度在过去的 20 年中持续下降, 只有 20 世纪 90 年代初数据的四分之一, 与 20 世纪 80 年代初相比 下降更大(图 2).

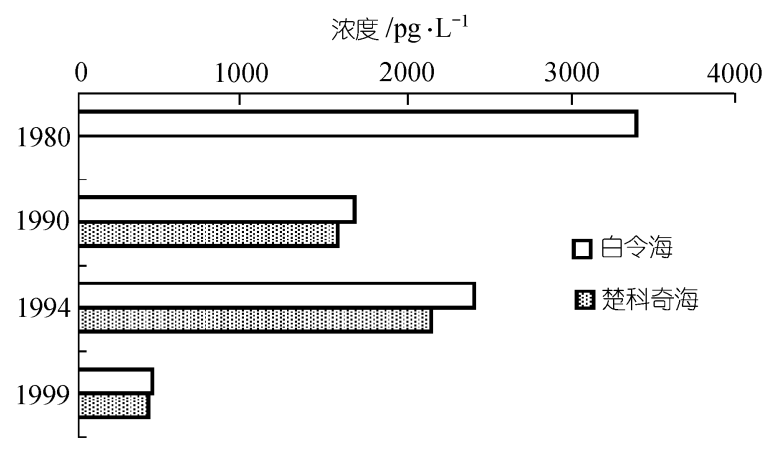

图 2 近 20 年来白令海及楚科奇海 $\mathrm{HCHs}$ 变化趋势 1980 年无楚科奇海数据

一般认为北半球的 OCPs 来源主要是中低纬度 的发展中国家农业上的使用, 然后由于大气输送及 海流作用而分布到全球. 本次考察发现白令海区在 北纬 $58^{\circ}$ 到 $59^{\circ}$ 之间存在一个 $\mathrm{HCHs}$ 的高浓度区域, 
其含量比两侧高 $20 \%$ 左右 (表 1). 从经度上考虑 $\mathrm{HCHs}$ 的总量从西向东逐渐减少, 但是趋势并不是十 分明显. 楚科奇海分布趋势类似, 西伯利亚海域的污 染状况要比阿拉斯加海域严重, 而且由于俄罗斯大 陆有机氯化合物污染较为严重 ${ }^{[10]}$, 导致离其较近的 $68^{\circ} \mathrm{N}$ 站位 $\mathrm{HCHs}$ 浓度比其他纬度都要高. 但是若将 两个海区作为一个整体进行比较, 污染状况并没有 显著的差别. 因为北极地区没有明显的 $\mathrm{HCHs}$ 来源, 该海区存在的有机氯农药应该是来自俄罗斯及北美 地区的环北极国家.

除了 HCHs 和 DDTs 之外, 我们在白令海及楚科 奇海地区发现了其他几种 OCPs. 例如: 艾氏剂、狄 氏剂和硫丹 I 等. 还发现在白令海峡南北两侧, 七氯 和环氧七氯表现出不同的分布特征, 在白令海地区 必须特别关注七氯的一种代谢物一一环氧七氯. 它 的检出率为 $54 \%$, 平均浓度为 $80.9 \mathrm{pg} \cdot \mathrm{L}^{-1}$; 而在楚 科奇海地区, 七氯的含量很高 $\left(94.6 \mathrm{pg} \cdot \mathrm{L}^{-1}\right)$, 检出率 为 $47 \%$, 这主要是由于楚科奇海地区的水温较低而 且表层水面上常年覆盖着大量的浮冰, 从而降低了 七氯向环氧七氯转化的速率; 另一方面也说明在北 极地区没有七氯的来源. 在检测的 16 种有机氯农药 中, 所有的站位都未检出 $\delta$-HCH, p, p'-DDD, 异狄氏 剂乙醛和硫丹硫酸盐. 白令海地区, 艾氏剂和硫丹 II 也在检出范围之下. 但是在楚科奇海, 艾氏剂和硫丹 II 的平均浓度分别为 77.6 和 $35.5 \mathrm{pg} \cdot \mathrm{L}^{-1}$, 检出率分 别为 $33 \%$ 和 $13 \%$. 因为没有这些污染物在低纬度的数 据, 所以很难判断这些物质的来源和途径.

(2) $\alpha / \gamma-\mathrm{HCH}$ 值. 1948 1997 年间全世界的 $\mathrm{HCHs}$ 产量估计为 1000 万吨. 一般工业产品中 $\mathrm{HCHs}$ 4 种异构体所占的比例是一定的: $\alpha: 55 \% \sim 80 \%, \beta$ : $5 \% \sim 14 \%, \gamma: 8 \% \sim 15 \%, \delta: 2 \% \sim 16 \%$. 而工业品林丹含 有 $90 \%$ 以上的 $\gamma \mathrm{HCH} . \alpha-\mathrm{HCH}$ 与 $\gamma \mathrm{HCH}$ 的比值常被用 来估计 $\mathrm{HCH}$ 的来源及其通过大气长距离输送的路 径 ${ }^{[4,5]}$. 从得到的数据分析, 白令海和楚科奇海的
OCPs 存在差异：白令海区的 $\alpha-\mathrm{HCH} / \gamma-\mathrm{HCH}$ 值在 5.0 左右，而楚科奇海在 1.9 3.8 之间，两者之间存在明 显差异. 这表明两个海区的 OCPs 的来源存在较大的 差异, 其中白令海区的 OCPs 是从低纬度地区大量使 用工业 $\mathrm{HCHs}$ 的地区经长途输运过来的, 而楚科奇海 区较低的 $\alpha-\mathrm{HCH} / \gamma-\mathrm{HCH}$ 值表明较近距离的污染源及 该地区周边存在着可能的林丹释放源.

与前人的数据相比, 白令海区 $\alpha-\mathrm{HCH} / \gamma-\mathrm{HCH}$ 值 大于 1988 年的结果(3.4), 而与 1993 年考察的结果 (4.8)类似. 这说明北极地区 $\alpha-\mathrm{HCH}$ 的相对浓度有上 升趋势, 但是, 关于这种趋势还没有得到公认的理论 解释. Pacyna 等人 ${ }^{[11]}$ 认为夏季 $\gamma-\mathrm{HCH}$ 可以通过光动 力学途径转化为 $\alpha-\mathrm{HCH}$; 而 Iwata 等 ${ }^{[2]}$ 则认为 $\alpha-\mathrm{HCH}$ 的相对升高是由于 $\gamma-\mathrm{HCH}$ 的亨利常数较低, 而易于 挥发所引起的.

\subsection{PCBs 的分布与组成}

自从 20 世纪 20 年代首次合成 PCBs 以来, 全世 界的 PCBs 总产量在几百万吨. 而环境介质中存在的 PCBs 一般是 135 种 PCBs 同类物组成的复杂混合 物 ${ }^{[12]}$. 该海区 PCBs 主要由四氯及五氯代 PCBs 同类 物组成, 其中包括持久性和疏水性最强的 CB118 和 CB153. 较低浓度的三氯及四氯 PCB 同类物表明大 气运输并不是该地区 PCBs 的主要来源 ${ }^{[13]}$. 在白令海, $\mathrm{CB} 101$ 和 $\mathrm{CB} 112$ 是主要的 PCBs 同类物, 占所选 PCBs 总量的一半; 而在楚科奇海, CB112, CB153 及 CB180 之和占所有 PCBs 的 60\%以上. 以前有结果表 ${ }^{\text {明 }}{ }^{[2]}$ : 北半球高浓度的 PCBs 主要分布在中低纬度, 但是由于各个国家对 PCBs 的生产和使用的限制逐渐 加强, 不同地区 PCBs 污染情况变得类似, 也就是说 高污染的地区含量降低, 而相对洁净地区 (如极 地)PCBs 含量有上升趋势.

低纬度的白令海区和较高纬度的楚科奇海区的 PCBs 总量没有明显的区别, 分别为 459.6 和 378.8

表 1 白令海和楚科奇海总 $\mathrm{HCHs}$ 平均含量及 $\alpha / \gamma-\mathrm{HCH}$ 值

\begin{tabular}{|c|c|c|c|c|c|}
\hline 白令海 & $\begin{array}{c}\mathrm{HCHs} \\
/ \mathrm{pg} \cdot \mathrm{L}^{-1}\end{array}$ & $\alpha / \gamma-\mathrm{HCH}$ & $\begin{array}{l}\text { 楚科 } \\
\text { 奇海 }\end{array}$ & $\begin{array}{c}\mathrm{HCHs} \\
/ \mathrm{pg} \cdot \mathrm{L}^{-1}\end{array}$ & $\alpha / \gamma-\mathrm{HCH}$ \\
\hline $56^{\circ} \mathrm{N}$ & 312.7 & 4.8 & $67^{\circ} \mathrm{N}$ & 225.5 & 1.9 \\
\hline $57^{\circ} \mathrm{N}$ & 491.8 & 5.7 & $68^{\circ} \mathrm{N}$ & 651.5 & 2.6 \\
\hline $58^{\circ} \mathrm{N}$ & 577.7 & 4.2 & $69^{\circ} \mathrm{N}$ & 285.3 & 3.8 \\
\hline $59^{\circ} \mathrm{N}$ & 580.0 & 5.5 & $70^{\circ} \mathrm{N}$ & 442.5 & 3.6 \\
\hline $60^{\circ} \mathrm{N}$ & 331.7 & 4.8 & $71^{\circ} \mathrm{N}$ & 460.0 & 3.0 \\
\hline $61^{\circ} \mathrm{N}$ & \multicolumn{2}{|c|}{ 456 ttips://engine.scichina.com/d } & $10.136728 \mathrm{~N}$ & 11523 & 3.7 \\
\hline
\end{tabular}


$\mathrm{pg} \cdot \mathrm{L}^{-1}$. 从组成上看, 并不是所有的 $\mathrm{PCBs}$ 同类物在 同一个站位中都能检出. 在白令海海域, CB52 和 CB180 是最普遍的 PCBs 同类物, 检出率约为 $80 \%$, 而在楚科奇海它们的检出率分别下降到 $60 \%$ 和 $44 \%$. 在大多数站位中, CB28 和 CB198 的浓度都接近或低 于检出限. 主要原因是 CB28 挥发性较强, 容易经过 海-气交换作用从表层海水重新挥发到大气中, 而且 它较易被生物富集 ${ }^{[14]}$. 由于高氯的 PCB 同类物 (如 CB198)挥发性较差, 很难从污染源向其他地区扩 散 ${ }^{[15]}$.

\section{4 重金属污染状况}

海洋中的重金属一般都有自然和人工两种来源. 澳大利亚附近开阔海域中溶解态锌、铅、镉的浓度估 计范围分别是：0.003 0.6, 0.001 0.04, 0.0001 0.12 $\mu \mathrm{g} \cdot \mathrm{L}^{-1}$, 但是很难区分它们的来源 ${ }^{[16]}$. 本研究用 ICP-MS 技术分析了白令海及楚科奇海区表层海水中 十几种痕量金属元素的总量. 结果证明, 白令海和楚 科奇海基本不存在重金属的污染，属于全球大洋背 景值范围 ${ }^{[17,18]}$. 与南极地区 (南极乔治王岛, 中国长 城站附近海域)表层海水中部分重金属含量也在同一 范围内(表 2).

表 2 北极与南极地区表层海水中部分重金属含量比较 $\left(\mathrm{ng} \cdot \mathrm{L}^{-1}\right)$

\begin{tabular}{rrrrr}
\hline 站位 $^{\text {a }}$ & \multicolumn{1}{c}{ 铅 } & \multicolumn{1}{c}{ 镉 } & \multicolumn{1}{c}{ 锡 } & \multicolumn{1}{c}{ 镓 } \\
\hline B1 & 115.3 & 180.8 & 56.0 & 84.5 \\
B2 & 316.0 & 175.0 & 56.0 & 145.6 \\
B3 & 63.2 & 135.9 & 43.6 & 125.4 \\
B4 & 200.5 & 87.8 & 52.1 & 92.8 \\
C1 & 144.8 & 68.2 & 38.0 & 53.0 \\
C2 & 114.8 & 83.1 & 35.4 & 100.1 \\
C3 & 226.1 & 121.3 & 57.4 & 142.8 \\
C4 & 137.1 & 89.6 & 139.3 & 59.0 \\
N1 & 72.5 & 144.4 & 128.2 & 104.5 \\
N2 & 173.6 & 218.0 & 138.4 & 159.4
\end{tabular}

a) B1 4 为白令海区; C1 4 为楚科奇海区; N1 2 为南极长城站 附近海区

在白令海的一个站位 $\left(178^{\circ} 48^{\prime} \mathrm{W}, 59^{\circ} 16^{\prime} \mathrm{N}\right)$ 采集 了分层 $(0,25,50,100,500,1000,1500 \mathrm{~m})$ 的重金属样 品. 图 3 为部分重金属(铅、镉、锡和镓)随深度的变 化情况. 从中可以看出, 铅、锡和镉在表层和底层海 水中的浓度基本一致. 而镓属于营养盐和生物共同
影响型元素 ${ }^{[19]}$, 在表层及混合层海水 $(0 \sim 150 \mathrm{~m})$ 中由 于生物吸收作用使浓度减少, 而在深海由于洋流和 生物地球循环的结果, 从颗粒物上重新释放而在底 层海水中富集, 所以其浓度随深度的变化最大, 底层 海水中的浓度是表层的 2 倍左右.

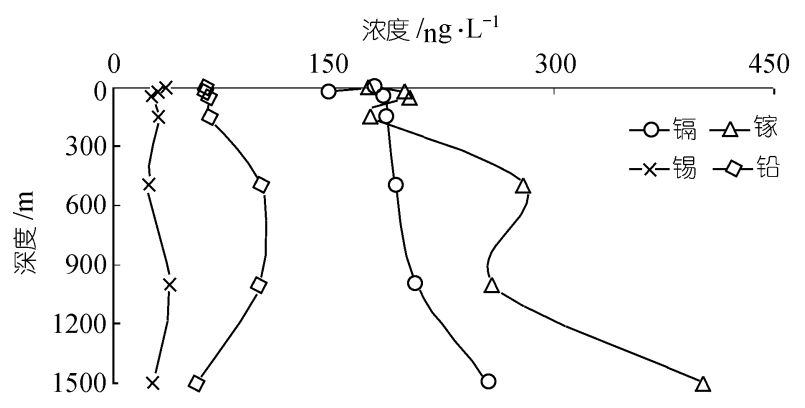

图 3 白令海某站位铅、镉、锡和镓随深度的变化

\section{3 结论}

$\alpha-\mathrm{HCH}$ ，七氯和环氧七氯是白令海及楚科奇海 表层海水最主要的 OCPs. $\alpha-\mathrm{HCH}$ 的浓度高于其他污 染物 1 2 个数量级, 与前人的考察结果相比, 含量自 1980 年持续下降, 而且自 20 世纪 90 年代中期以来下 降速度加快. 该地区的重金属含量属于典型较洁净 的大洋环境, 未存在重金属污染. 典型重金属垂直变 化趋势不同. 由于条件所限，本次考察研究介质单一， 要完整了解北极地区环境状况仍需要进行多介质深 人研究.

致谢 感谢国家海洋局极地考察办公室中国首次北极科 学考察队和北京师范大学赵俊林教授及国家海洋环境监 测中心李洪、周传光高级工程师的帮助. 本工作为中国首 次北极科学考察及中国科学院创新工程(KZCX2-414)资助 项目.

\section{参考文献}

1 Atlas E, Giam C S. Global transport of organic pollutants: ambient concentrations in the remote marine atmosphere. Science, 1981, 211: $163 \sim 165$

2 Iwata $\mathrm{H}$, Tanabe $\mathrm{S}$, Sakai N, et al. Distribution of persistent organochlorines in the oceanic air and surface seawater and the role of ocean on their global transport and fate. Environ Sci Technol, 1993, 27: $1080 \sim 1098$

3 Tanabe S, Mori T,5Tatsukawa R. Global pollution of marine 
mammals by PCBs, DDTs and $\mathrm{HCHs}(\mathrm{BHC}) \mathrm{s}$. Chemosphere, 1983, 12: $1269 \sim 1275$

4 Kawano $\mathrm{M} \mathrm{T}$, Inoue $\mathrm{T}$, Wada $\mathrm{H}$, et al. Bioconcentration and residue patterns of chlordane compounds in marine animals: invertebrates, fish, mammals, and seabirds. Environ Sci Technol, 1988, 22: $792 \sim 797$

5 Hinckley D A, Bidleman T F, Rice C P. Atmospheric organochlorine pollutants and air-sea exchange of hexachlorocyclohexane in the Bering and Chukchi Seas. J Geophys Res, 1991, 96: $7201 \sim 7213$

6 Chernyak S M, McConnell L L, Rice C P. Fate of some chlorinated in Arctic and far eastern ecosystems in the Russian Federation. Sci Total Environ, 1995, 160: $75 \sim 85$

7 Jantunen L M, Bidleman T F. Reversal of the air-water gas exchange direction of hexachlorocyclohexanes in the Bering and Chukchi Sea: 1993 versus 1988. Environ Sci Technol, 1995, 29: $1081 \sim 1089$

8 Jantunen L M, Bidleman T F. Organochlorine pesticides and enantiomeers of chiral pesticides in Arctic Ocean water. Arch Environ Contam Toxicol, 1998, 35: $218 \sim 228$

9 Fujishima Y, Ueda K, Maruo M, et al. Distribution of trace bioelements in the subarctic North Pacific Ocean and the Bering Sea (the R/V Hakuho Maru Cruise KH-97-2). J Oceanogr, 2001, 57: $261 \sim 273$

10 Fedorov L A. Persistent organic chemicals in the former Soviet Union. Environ Poll, 1999, 105: 283 287

11 Pacyna $\mathbf{J}$ M, Oehme M. Long-range transport of some organic compounds to the Norwegian Arctic. Atmos Environ, 1998, 22:
$243 \sim 257$

12 Ballschmiter K, Zell M. Analysis of polychlorinated biphenyls (PCB) by glass capillary gas chromatography. Fresenius Z Anal Chem, 1980, 302: $20 \sim 31$

13 Henriksen E O, Gabrielsen G W, Trudeau S, et al. Organochlorines and possible biochemical effects in glaucous gulls (Larus hyperboreus) from Bjornoya, the Barents sea. Arch Environ Contam Toxicol, 2000, 38: $234 \sim 243$

14 Montone R C, Taniguchi S, Sericano J, et al. Determination of polychlorinated biphenyls in Antarctic macroalgae Desmarestia sp Sci Total Environ, 2001, 277: $181 \sim 186$

15 Sweetman A J, Jones K C. Declining PCB concentrations in the UK atmosphere: evidence and possible causes. Environ Sci Technol, 2000, 34: $863 \sim 869$

16 Bruland K W, Donat J R, Hutchins D A. Interactive influences of bioactive trace metals on biological production in oceanic waters. Limnology Oceanog, 1991, 36: 1555 1577

17 Danielsson L G, Magnusson B, Westerlund S. Cadmium, copper, iron, nickel and zinc in the North-East Atlantic Ocean. Mar Chem 1985, 17: $23 \sim 41$

18 Haroldsson C, Westerlund S. Trace metals in the water columns of the Black Sea and Frmvaren Fjord. Mar Chem, 1988, 23: 417 424

19 Li Y H. Distribution patterns of the elements in the ocean: a synthesis. Geochim Cosmochim Acta, 1991, 55: 3223 3240

(2002-04-05 收稿, 2002-05-29 收修改稿) 